\title{
Two-Dimensional Liquid Polymer Diffusion: Experiment and Simulation
}

\author{
Uwe Albrecht, Achim Otto, and Paul Leiderer \\ Fakultät für Physik, Unicersität Konstanz, D-7750 Konstanz, Germany
}

(Received 21 January 1992)

\begin{abstract}
The spreading profiles of small (picoliter) droplets of polydimethylsiloxane on silver surfaces have been monitored in the thickness range below one monolayer. The profile shapes are distinctly nonGaussian and can be approximated rather well by spherical caps. The experimental shapes and spreading dynamics are compared with Monte Carlo simulations of spreading polymer liquids. Excellent agreement is found.

PACS numbers: $68 \cdot 15 .+\mathrm{e}, 47.15 .-\mathrm{x}$
\end{abstract}

The dynamics of wetting and spreading of molecular liquids on solid surfaces has been a topic receiving increasing attention in the past few years. There have been a number of experiments recently probing droplet shapes [1-5] and capillary rise profiles [1,2] as well as investigations of the structure of the precursor film $[4,6]$ that precedes the advancing of the macroscopic edge of the liquid. Experimental techniques included ellipsometry $[1,3,4]$, interferometry [4-6], and x-ray reflectivity [2,4]. There have also been molecular dynamics simulations probing contact angles [7] and terraced spreading [8]. Theoretical investigations $[9,10]$ have focused on phenomena in the thick film regime, where a continuum description is appropriate and collective effects are involved. As yet no good theory for the behavior of the outer spreading edge of monolayer and submonolayer thickness is available. Even though this thickness range is well resolved in some experiments, there is only one systematic study so far undertaken by Heslot, Cazabat, and Fraysse [1] who measured the width of the spreading edge as a function of time. Further studies of this very thin film spreading behavior are needed to provide a better understanding of the effects involved there, which might play a crucial role in the spreading dynamics of the entire system.

In this Letter we present measurements of the profiles of very small liquid drops with volumes in the picoliter range of the polymer polydimethylsiloxane (PDMS) deposited on a silver surface. The investigations concentrate on the temporal development of the profile at times where due to spreading the maximum thickness in the droplet center has dropped to less than a molecular monolayer. The experimental results are then compared to a Monte Carlo study of a two-dimensional polymer system.

The droplet profiles were measured using optically excited surface plasmons. Details of the technique are described elsewhere [11]. We have used the so-called Kretschmann configuration [12], where a thin silver film, evaporated onto the base of a glass prism, is illuminated by a light beam (in our case from a HeNe laser) from the glass side. As the angle of incidence $\theta$ is scanned, a sharp drop in the reflected intensity is observed at a particular value $\theta_{R}$, arising from the resonant excitation of surface plasmons on the silver film. A thin dielectric layer on the silver changes the surface plasmon resonance condition, the shift of the incidence angle at which resonance occurs (compared to the uncovered surface) gives the layer thickness $d$. By monitoring the resonance angle $\theta_{R}$ accurately we can determine the film thickness of the dielectric layer with sub-angstrom resolution. The lateral resolution, achieved by scanning of the incident laser beam, was $120 \mu \mathrm{m}$, given by the laser spot diameter.

The silver films, serving as the substrate both for the PDMS and for exciting the surface plasmons, were prepared by thermal evaporation to a thickness of $530 \AA$. The typical roughness scale of such films, as determined by scanning tunneling microscopic (STM) inspection, was a few $100 \mathrm{~nm}$ parallel and some $10 \mathrm{~nm}$ perpendicular to the film plane. The PDMS used here was of commercial grade (Aldrich, No. 31766-7, mean molecular weight $M_{w}=760$, viscosity $\eta=4.6 \times 10^{-3} \mathrm{Pas}$, surface tension $\sigma=0.021 \mathrm{~N} / \mathrm{m}$ ). We deposited the PDMS droplets shortly after preparing the Ag films. The experiments were performed under air in a closed box to avoid contamination by dust.

Figure 1 shows the thickness profile evolution of a droplet with $\sim 6 \mathrm{pl}$ volume. The earliest profile, taken about 15 min after deposition, displays a thickness in the droplet center still larger than a monolayer. An indication for a monomolecular foot (as observed in other spreading studies of PDMS as well [3]) can be seen. Later profiles have central thicknesses equal to or even smaller than a monolayer. (It should be pointed out that at such a small thickness the picture of a continuum droplet breaks down, and the ordinates in Fig. 1 should be viewed more accurately as an areal density profile of the polymer molecules of the surface; nevertheless, we will refer to these density profiles as "droplets" for simplicity.) The slight asymmetry of the first profile is due to the spreading of the PDMS during the lateral scan.

Figure 2 shows best fits of a Gaussian and a spherical cap with one of the profiles with submonolayer thickness. Evidently it cannot be described satisfactorily by a Gaussian, whereas a spherical cap accounts very well for the droplet shape. This holds for all measured droplets 


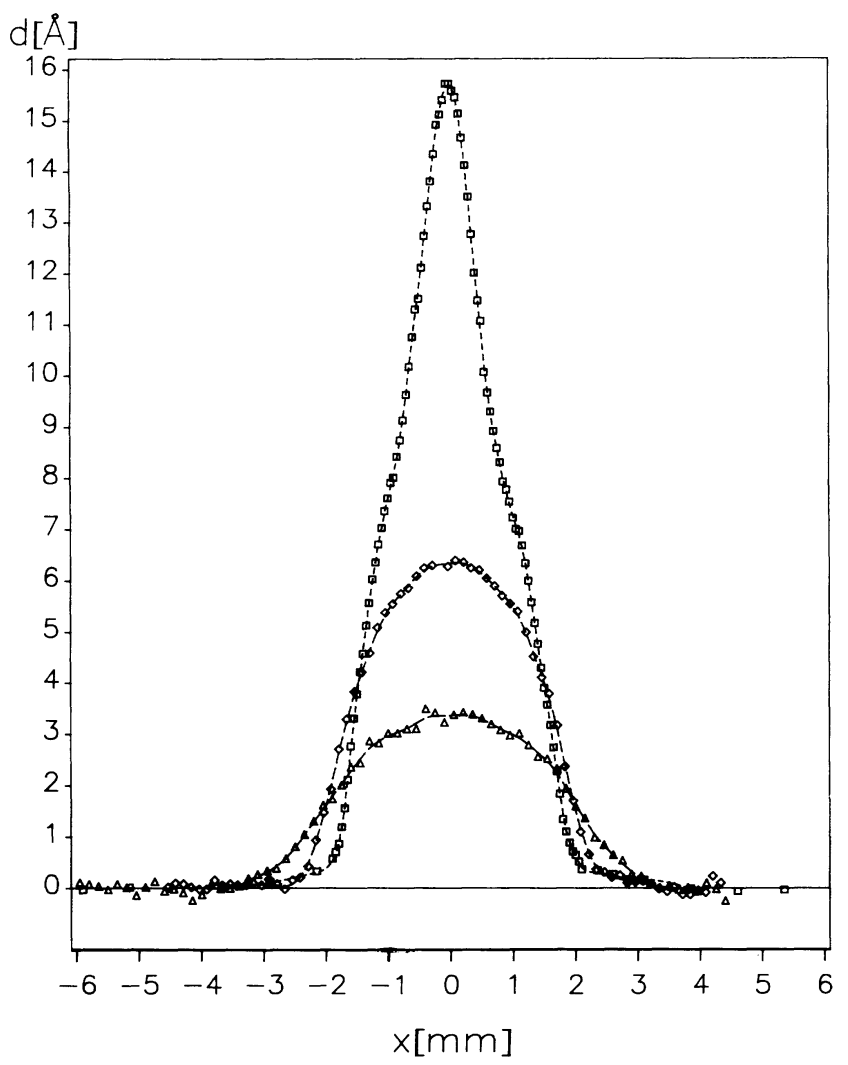

FIG. 1. Thickness profiles of a PDMS drop (6 pl volume) 35 , 80 , and $360 \mathrm{~min}$ after deposition.

(looking more closely, a small smeared out foot can be seen at the edge of the spherical cap shape, which grows towards longer spreading times; it is referred to later). This result is surprising at the first glance since a freely diffusing two-dimensional surface gas is expected to develop a Gaussian density distribution. On closer inspection, however, this apparent discrepancy is resolved, because the motion of polymer molecules in a film of approximately monolayer thickness may differ considerably from the diffusion of pointlike particles. In fact, the polymer chains distributed across the surface will partly block each other, leading to a hindered diffusion in the dense central region of the droplet. Only in the dilute region with an average thickness far below one monolayer, i.e., when the polymer molecules are far apart, the usual diffusion process should take over. This behavior can equivalently be described by a concentration-dependent diffusion coefficient. There the mutual blocking of polymers at high areal densities is accounted for by an effective diffusion coefficient, which is different for each concentration.

Additional evidence that one is not dealing here with simple diffusion of independent molecules is obtained from the temporal evolution of the droplet radius $b$. As an example, we have plotted in Fig. 3 the radii of two droplets versus time on a double logarithmic scale. As

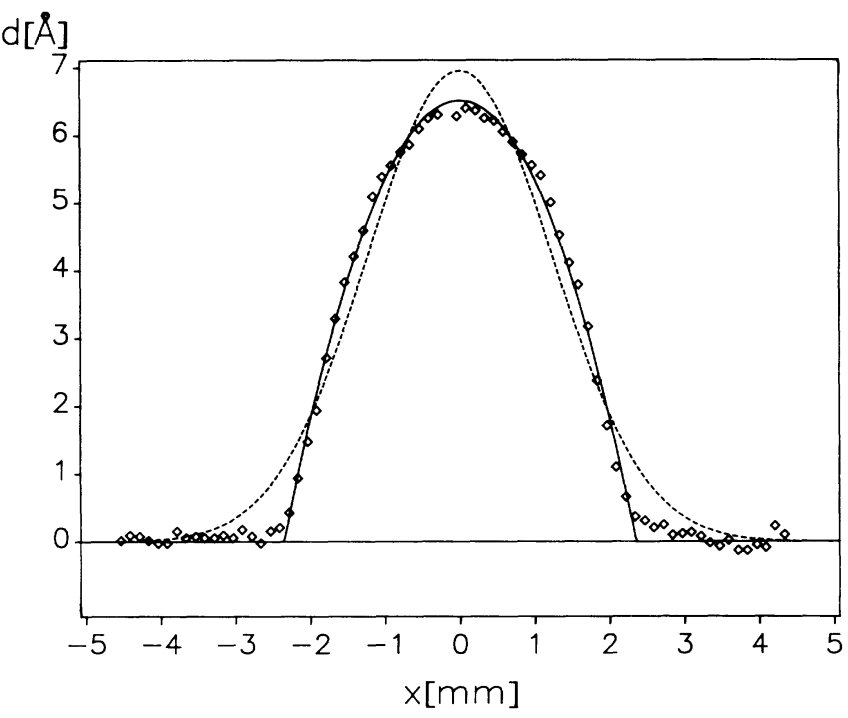

FIG. 2. Fits of a Gaussian (dashed line) and a spherical cap (solid line) with the middle profile shown in Fig. 1. It is fitted very well by a spherical cap, whereas a Gaussian cannot account for the droplet shape.

suggested by the straight lines the growth roughly follows a law $r(t) \sim t^{\alpha}$, with growth exponents $\alpha$ around 0.13 . By contrast, a spreading process dominated by free diffusion is expected to be described by $\alpha=0.5$, a value distinctly larger than that observed in the experiment. Thus we conclude also from this result that the diffusion of the polymer molecules must be severely restricted.

In order to corroborate the above qualitative arguments by a microscopic model and to clarify the role of the processes involved we have performed Monte Carlo simulations of two-dimensional polymer diffusion. For this purpose an algorithm analogous to the bond fluctuation method introduced by Carmesin and Kremer [13] was used employing a single-site model on a $600 \times 600$

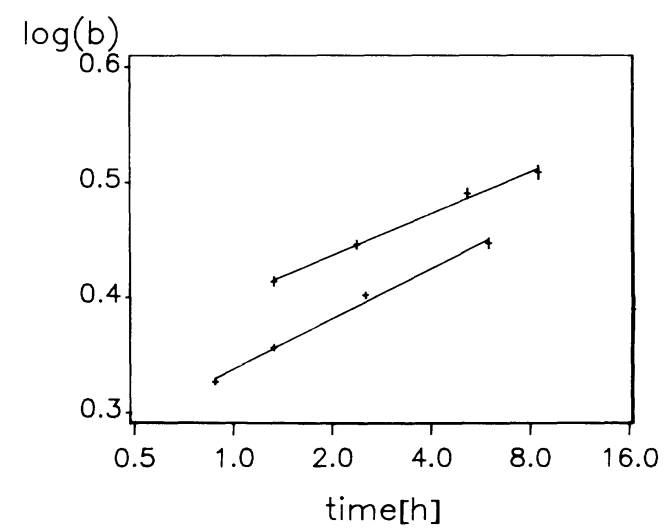

FIG. 3. Double logarithmic plot of drop radius $b$ vs spreading time for two different PDMS droplets. The straight lines assume a growth law $b \sim t^{a}$, giving $\alpha=0.12$ and 0.14 for the droplets shown. 
(a)

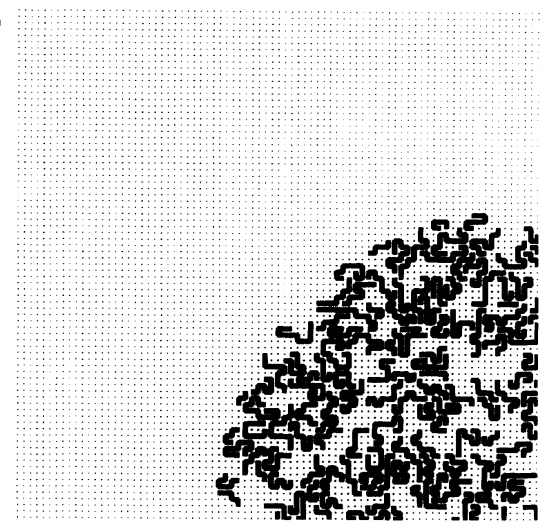

(b)

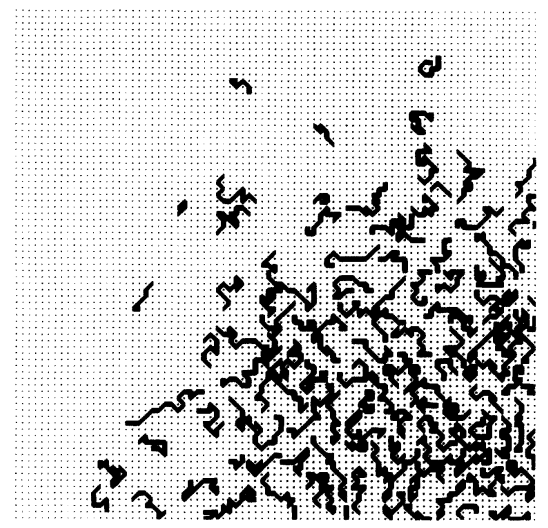

FIG. 4. Closeup section of a polymer configuration used in the simulations. One quadrant of the simulated droplet is shown. (a) Starting configuration produced by random growth of polymers (only right angles are allowed here to avoid blocked configurations). (b) Same simulation after 2000 Monte Carlo steps (MCS) per monomer. Small dots indicate lattice sites.

square lattice (blocked configurations were excluded). We simulated self-avoiding walks starting from a "pancake" areal density distribution (the final stage of a spreading droplet in the model of de Gennes [10]). The starting polymer configuration was achieved by growing the polymers at random from evenly distributed fixed lattice sites in a self-avoiding way allowing only a limited number of growth attempts. This procedure, quite analogous to experimental polymer growth, naturally yields a distribution of polymer lengths. Figure 4 shows a closeup of the areal polymer distribution of one quadrant of the simulated droplet at different times. The entanglement is clearly seen to remain in the central part also at later stages of the spreading process, whereas the outer polymers get progressively free.

Profiles obtained from distributions as in Fig. 4 are plotted in Fig. 5(a) for various stages of the simulation. The polymer length distribution used in the runs shown peaked at a length of 10 (close to the PDMS used in the experiments) with a half-width of six monomers. The shapes of the simulated profiles are remarkedly similar to the experimental shapes at comparable stages of the spreading displayed in Fig. 5(b). The simulated as well as the experimental data again agree very well with spherical caps. The hindered diffusion, on which the simulation is based, therefore appears to account very

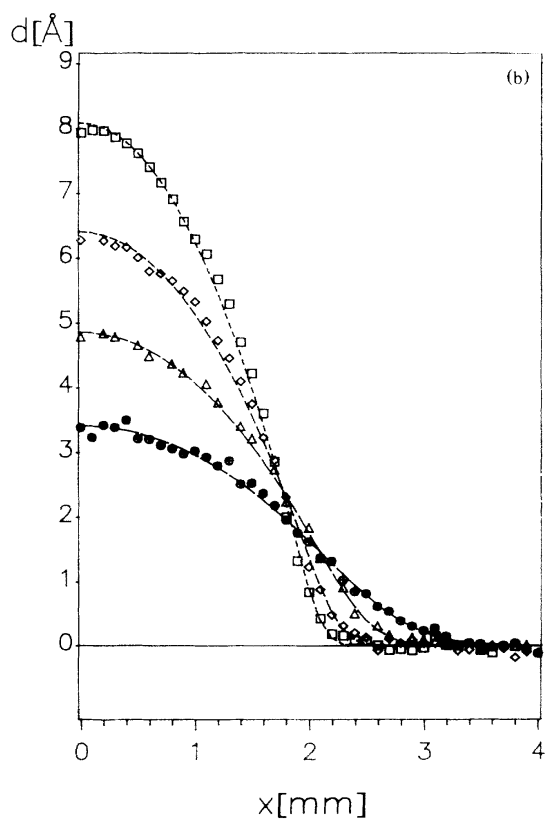

FIG. 5. (a) Simulated droplet profiles at $2000,4000,8000$, and $18000 \mathrm{MCS} /$ monomer. The thickness $d$ is given in percent of occupied lattice sites (a typical starting configuration has slightly above $60 \%$ coverage). (b) Experimentally determined PDMS droplet profiles at comparable stages of the spreading process, 50,80, 150, and $360 \mathrm{~min}$ after deposition. The dashed lines are fitted spherical caps convoluted with narrow Gaussians to account for the smeared out foot. 
well for the experimental droplet shapes. The fits shown in Fig. 5 include a convolution with a narrow Gaussian to account for the smeared out foot of the caps. This foot, growing with time, reflects the different behavior of the polymers in the region, where the mean distance between two molecules is larger than their average extension. In this case the chains do not block each other anymore and free diffusion of then independent molecules dominates. This picture is supported by the growth exponent of the width of the Gaussian foot being 0.5 as expected for free diffusion. The size of the foot thus should depend on the length of the molecules, with shorter chains leading to a longer foot. This is indeed confirmed by using different polymer lengths in the simulations [14].

We compared the dynamics of the measured polymer spreading and the simulation by looking at the growth exponents for the width $b$ and the thickness $d$. We found $b \sim t^{0.2}$ and $d \sim t^{-0.45}$ in the simulation, compared to exponents around 0.13 for $b$ and -0.41 for $d$ in the experiments (although both in the simulations as in the experiments there are indications for different growth regimes; i.e., a description by one simple growth law $\sim t^{\alpha}$ may not be that appropriate). The fact that the growth in the measured droplets is slightly slower than in the simulations might reflect an influence of the surface roughness of the silver substrate. Very large roughness (distinctly larger than in our case) has been shown to slow down the spreading process [15]. It could also be an effect of the relatively small size of the simulated system, that might lead to slightly faster dynamics.

In summary, we have presented both measurements and simulations of the time evolution of polymer droplet profiles at submonolayer thickness. These droplets can be viewed as two-dimensional polymer liquids. Monte Carlo calculations yield the experimentally observed spherical cap shapes of the profiles and even reproduce the spreading dynamics quite well. This result also lends support to the validity of the bond fluctuation method [13] used in the simulation. The behavior is exclusively due to the polymeric nature of the particles involved and is quite different from that of rigid particles. These polymeric effects should be taken into account when modeling spreading behavior of polymer substances, which plays a dominant role in all lubrication processes.

This work was supported by the SFB 306 of the Deutsche Forschungsgemeinschaft.

[1] F. Heslot, A. M. Cazabat, and N. Fraysse, J. Phys. Condens. Matter 1, 5793 (1989).

[2] J. Daillant et al., Europhys. Lett. 6, 431 (1988); J. Daillant, J. J. Benattar, and L. Leger, Phys. Rev. A 41, 1963 (1990).

[3] F. Heslot, A. M. Cazabat, and P. Levinson, Phys. Rev. Lett. 62, 1286 (1989); F. Heslot, N. Fraysse, and A. M. Cazabat, Nature (London) 338, 640 (1989); F. Heslot et al., Phys. Rev. Lett. 65, 599 (1990).

[4] L. Leger et al., Rev. Phys. Appl. 23, 1047 (1988).

[5] J. D. Chen and N. Wada, Phys. Rev. Lett. 62, 3050 (1989); P. Silberzan and L. Leger, Phys. Rev. Lett. 66, 185 (1991).

[6] D. Beaglehole, J. Phys. Chem. 93, 893 (1989).

[7] J. Hautman and M. L. Klein, Phys. Rev. Lett. 67, 1763 (1991).

[8] J. Yang, J. Kopnik, and J. R. Banavar, Phys. Rev. Lett. 67, 3539 (1991).

[9] J. F. Joanny and J. P. de Gennes, J. Phys. (Paris) 47, 121 (1986); A. M. Cazabat, Contemp. Phys. 28, 347 (1987); N. V. Churaev, Rev. Phys. Appl. 23, 975 (1988); D. B. Abraham et al., Phys. Rev. Lett. 65, 195 (1990); A. M. Cazabat et al., J. Phys. Chem. 94, 7581 (1990).

[10] P. G. de Gennes, Rev. Mod. Phys. 57, 827 (1985).

[11] U. Albrecht, H. Dilger, P. Evers, and P. Leiderer, in Process Module Metrology, Control and Clustering, edited by C. J. Davis, T. R. Turner, and I. P. Herman, SPIE Proc. No. 1594 (SPIE, Bellingham, 1992).

[12] E. Kretschmann and H. Raether, Z. Naturforsch. 23a, 2135 (1968).

[13] I. Carmesin and K. Kremer, Macromolecules 21, 2819 (1988); J. Phys. (Paris) 51, 915 (1990).

[14] We also performed simulations with a shorter polymer distribution, peaking at a length of 6 monomers, and with a set of polymers all of them having fixed length 12 . Apart from the changes in the size of the Gaussian foot the spreading behavior turned out to be essentially the same.

[15] A. M. Cazabat and M. A. Cohen Stuart, J. Phys. Chem. 90, 5845 (1986). 
(a)

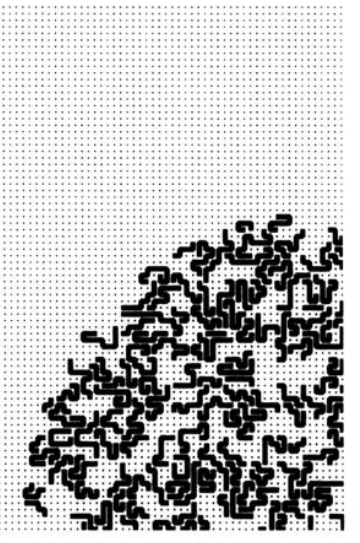

(b)

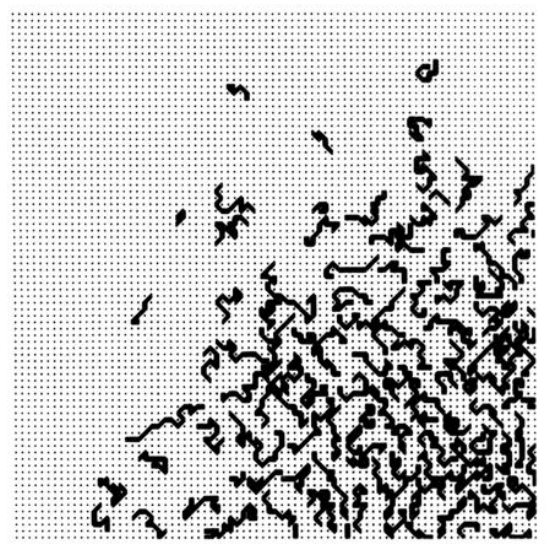

FIG. 4. Closeup section of a polymer configuration used in the simulations. One quadrant of the simulated droplet is shown. (a) Starting configuration produced by random growth of polymers (only right angles are allowed here to avoid blocked configurations). (b) Same simulation after 2000 Monte Carlo steps (MCS) per monomer. Small dots indicate lattice sites. 